\title{
Automatic Text Summarization Using Two-Step Sentence Extraction
}

\author{
Wooncheol Jung ${ }^{1}$, Youngjoong $\mathrm{Ko}^{2}$, and Jungyun $\mathrm{Seo}^{1}$ \\ ${ }^{1}$ Computer Science, Sogang University, Sinsu-dong 1, Mapo-gu \\ Seoul, 121-742, Korea \\ wcjung@nlprep.sogang.ac.kr, seojy@ccs.sogang.ac.kr \\ ${ }^{2}$ Division of Electrical, Electronics, and Computer Engineering, Dong-A University, \\ 840 Hadan 2-dong, Saha-gu, Busan, 604-714, Korea \\ yjko@daunet.donga.ac.kr
}

\begin{abstract}
Automatic text summarization sets the goal at reducing the size of a document while preserving its content. Our summarization system is based on Two-step Sentence Extraction. As it combines statistical methods and reduces noise data through two steps efficiently, it can achieve high performance. In our experiments for $30 \%$ compression and $10 \%$ compression, our method is compared with Title, Location, Aggregation Similarity, and DOCUSUM methods. As a result, our method showed higher performance than other methods.
\end{abstract}

\section{Introduction}

The goal of text summarization is to take an information source, extract content, and present the most important content to a user in a condensed form and in a manner sensitive to the user's or application's needs [10]. To achieve this goal, text summarization system should identify the most salient information in a document and convey it in less space than original text. The most widespread summarization strategy is still sentence extraction. Traditional text summarization methods have used linguistic approaches and statistical approaches to extract salient sentences. But some problems have occurred in both methods for text summarization. Despite high performance, linguistic approaches have some difficulties in requiring to use high quality linguistic analysis tools such as discourse parser and linguistic resources such as WordNet, Lexical Chain, and Context Vector Space [1][7][12]; they are very useful resources for summarization systems but a weak point of them is to take much time and high cost to construct. On the other side, statistical approaches are easy to understand and implement, but generally they show low accuracy.

In this paper, we propose a new high performance summarization method to efficiently combine statistical approaches. By combining several statistical methods in two steps, our method can obtain higher accuracy than other statistical methods or the linguistic method (DOCUSUM) [7]. Moreover, our method can also have low cost and robust system architecture because it does not require any linguistic resources.

In the first step of our system, our method creates bi-gram pseudo sentences by combining two adjacent sentences for solving feature sparseness problem; it occurs in

S. H. Myaeng et al. (Eds.): AIRS 2004, LNCS 3411, pp. 71-81, 2005.

(C) Springer-Verlag Berlin Heidelberg 2005 
text summarization because of using only one sentence as the linguistic unit. And then our method applies the combined statistical method (Title and Location) to the bigram pseudo sentences for calculating the importance of them. The goal of the first step is not to extract salient sentences but to remove noisy sentences. Thereafter, we can get more useful pseudo sentences through removing noisy pseudo sentences. Then, in the second step, our method separates the bi-gram pseudo sentences into each original single sentence and it performs second sentence extraction by adding Aggregation Similarity method [5] to the linear combination of the first step. Because the Aggregation Similarity method estimates the importance of sentences by calculating the similarities of all other sentences in a document, it can be more efficient in our system after removing the noisy sentences. Since our system carries out a summarization task without any linguistic resources such as WordNet and discourse parser, it could be low cost and robust. As shown in experimental results, our system showed higher performance than other statistical methods and DOCUSUM as a linguistic method.

The rest of this paper is organized as follows. Section 2 describes related works in existing summarization systems. In Section 3, we present the methods used in each step in detail. Section 4 is devoted to evaluating experimental results. In the last section, we draw conclusions and present future works.

\section{Related Works}

The summarization system has two main categories: Linguistic approaches and Statistical approaches. The former uses semantic relations between words, phrase, and clause structural information in a sentence by using the linguistic resources while the latter uses title, frequency, location, and cue words and so on.

\subsection{Linguistic Approaches}

Bazilay and Elhadad constructed Lexical Chain by calculating semantic distance between words using WordNet [1]. Strong Lexical Chains are selected and the sentences related to these strong chains are chosen as a summary. The methods which use semantic relations between words depend heavily on manually constructed resources such as WordNet [12]. WordNet is not available in several languages such as Korean and this kind of linguistic resources are hard to maintain.

To overcome the limitation of this problem, Ko, Kim, and Seo constructed Lexical Clusters [7]. Each Lexical Cluster has different semantic categories while they are more loosely connected than Lexical Chains. They call their system DOCUSUM.

Marcu used discourse structural information [11]. This is based on contextual structure through analyzing sentence relations and sentence semantics.

These Linguistic approaches are producing high quality summary but, in case of time and expansion manner, they leave much room for improvement.

\subsection{Statistical Approaches}

The pioneering work studied that most frequent words represent the most important concepts of the text [8]. This representation abstracts the source text into a frequency 
table. Therefore, this method ignores the semantic content of words and their potential membership in multi-word phrases.

In other early summarization system, Edmundson studied that first paragraph or first sentences of each paragraph contain topic information [2]. Also he studied that the presence of words such as significant, hardly, impossible signals topic sentences.

A query-based summarization makes a summary by extracting relevant sentences from a document [3]. The criterion for extraction is given as a query. The probability of being included in a summary increases according to the number of words cooccurred in the query and a sentence.

\section{Two-Step Sentence Extraction}

\subsection{Overall Architecture}

Our summarization system is based on statistical approaches. Generally, they have several weak points: feature sparseness and low performance. The former problem is caused by extracting features from only one sentence and the latter is caused by depending on the particular format and the style of writing.

In order to successfully deal with the feature sparseness problems, we made an assumption. The salient sentences are grouped at a definite position without regarding to location of subjective sentences. To apply it to our system, we combine two adjacent sentences into bi-gram pseudo sentence. This extension of semantic unit can resolve the feature sparseness problem in part.

In order to improve the performance of statistical methods regardless of the particular formats, we make an efficient combination of them by means of estimating various statistical methods.

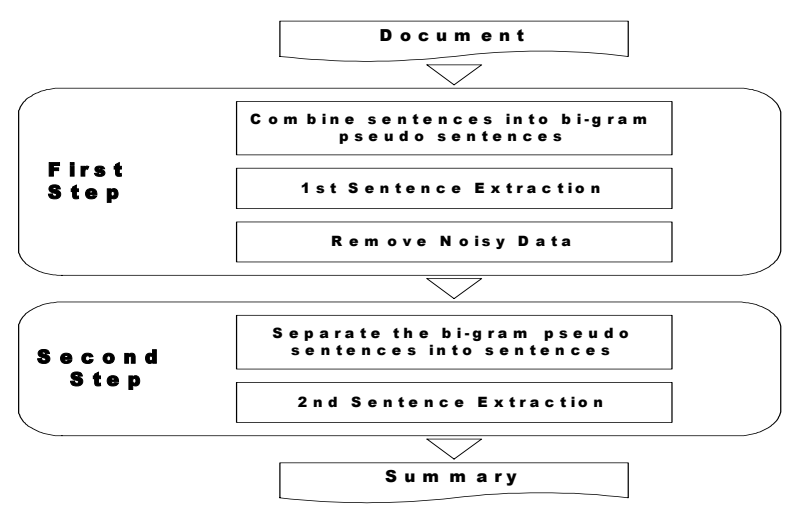

Fig. 1. Illustration of our summarization system

In the first step, the system estimates the importance score of bi-gram pseudo sentences by the combination of Title and Location methods. And then it removes invaluable bi-gram pseudo sentences which are called by noisy data. In the second step, 
it extracts salient sentences for summary from original single sentences which are separated from remaining bi-gram pseudo sentences. Here, we add Aggregation Similarity method to our combination method to achieve better performance.

The following Fig. 1 shows the architecture of our summarization system.

\subsection{General Statistical Methods}

Our system uses several statistical methods. We here present several typical statistical methods.

Title Method. The score of sentences is calculated as how many words are commonly used between the sentence and title. This calculation is acquired by a query from title in Boolean weighted vector space model. The inner product method is exploited for similarity between a sentence and a query.

$$
\begin{gathered}
\operatorname{sim}\left(S_{i}, Q\right)=\sum_{k=1}^{n} w_{i k} w_{q k} . \\
\operatorname{Score}\left(S_{i}\right)=\operatorname{sim}\left(S_{i}, Q\right) .
\end{gathered}
$$

where $S_{i}$ is an $i$-th sentence and $Q$ is a title query. And $w_{i k}$ is the weight of $k$-th word in $i$-th sentence and $w_{q k}$ is the weight of $k$-th word in the query.

Location Method. It has been said that the leading several sentences of an article are important and a good summary [15]. Therefore, the leading sentences in compression rate are extracted as a summary by the location method.

$$
\operatorname{Score}\left(S_{i}\right)=1-\frac{i-1}{N} .
$$

where $S_{i}$ is an $i$-th sentence and $N$ is the total number of sentences in the text.

Aggregation Similarity Method. The score of a sentence is calculated as the sum of similarity with other all sentence vectors in document vector space model. Each score is computed as follows [5].OP

$$
\begin{gathered}
\operatorname{sim}\left(S_{i}, S_{j}\right)=\sum_{k=1}^{n} w_{i k} w_{j k} . \\
\operatorname{Score}\left(S_{i}\right)=\operatorname{asim}\left(S_{i}\right)=\sum_{j=1, j \neq i}^{m} \operatorname{sim}\left(S_{i}, S_{j}\right) .
\end{gathered}
$$

Equation 4 calculates the similarity with other sentence and $w_{i k}$ is the weight of $k$ th word in $i$-th sentence.

Frequency Method. The frequency of term occurrences within a document has often been used for calculating the importance of sentences [14]. In this method, the score of a sentence can be calculated as the sum of the score of words in the sentence. The score of important score $w_{i}$ of word $i$ can be calculated by the traditional tf.idf method as follows [13]. 


$$
w_{i}=t f_{i} \times \log \frac{N}{d f_{i}} .
$$

where $t f_{i}$ is the term frequency of word $i$ in the document, $N$ is the total number of texts, and $d f_{i}$ is the document frequency of word $i$ in the whole data set.

\subsection{The TF-Based Query Method}

As described above, the title has usually been used for a query and the Title method has shown higher performance than other approaches in general. However, in special cases, it can be hard to extract a title from documents or any style of documents has no-title. For these cases, we propose a method to extract topic words for a query. The TF-based query method uses a query which consists of words with the highest term frequency in a document. This method considers words with high frequency as important concepts such as [8].

Like the Title method, the inner product metric is used as the similarity measure between a sentence and a TF-based query. To represent sentences, only proper and common nouns are used after eliminating stop words. For sentence vectorization, the Boolean weighting is used as follows:

$$
\begin{aligned}
& S_{i}=\left(w_{i 1}, w_{i 2}, w_{i 3}, \ldots, w_{i n}\right) \\
& w_{i k}=\left\{\begin{array}{ll}
1 & \text { if } \mathrm{tf}_{i k}>0 \\
0 & \text { otherwise }
\end{array} .\right.
\end{aligned}
$$

where $t f_{i k}$ is the term frequencies of $k$-th word in sentence $i$ and $S_{i}$ is sentence vector. In general, $t f . i d f$ representation has shown better performance in information retrieval. However, binary representation has generally showed better performance in summarization [4].

By the following Equation 7, we calculate the similarity between sentences and the TF-based query.

$$
\operatorname{sim}\left(S_{i}, T f Q\right)=\sum_{k=1}^{n} w_{i k} w_{T F Q k} .
$$

where $n$ is the number of words which is included in a document. $w_{i k}$ is the weight of $k$-th word in $i$-th sentence and $w_{T F Q k}$ is the weight of $k$-th word in the TF-based query.

To verify our TF-based query method, we did experiments according to the number of topic words as shown in Table 1 . As a result, we achieved the best performance when using one topic word, the most frequent word.

Table 1. Performances according to the number of word

\begin{tabular}{lcc}
\hline & $30 \%$ & $10 \%$ \\
\hline 1 Topic Word & 0.500 & 0.526 \\
2 Topic Words & 0.490 & 0.434 \\
3 Topic Words & 0.478 & 0.367 \\
4 Topic Words & 0.455 & 0.330 \\
5 Topic Words & 0.450 & 0.313 \\
\hline
\end{tabular}




\subsection{The Combination of Statistical Methods in Two Steps}

In this section, we describe a new combinational method for statistical approaches. Before introducing our method in detail, we observed the performance of each statistical method to choose statistical methods used in our combinational method. The performance of general statistical methods are shown in Table 2.

Table 2. Experimental results of statistical methods

\begin{tabular}{ccc}
\hline Method & $30 \%$ & $10 \%$ \\
\hline Title & 0.488 & 0.435 \\
Location & 0.494 & 0.466 \\
TF based query & 0.456 & 0.465 \\
Aggregation Similarity & 0.406 & 0.239 \\
Frequency & 0.352 & 0.130 \\
\hline
\end{tabular}

Our combinational method exploits Title, Location, Aggregation Similarity methods as considering their performances and characteristics. For a case of no-title documents, the TF-based method is used instead of the Title method.

\subsubsection{Removing the Noisy Sentences in the First Step}

In the first step, our goal is to reduce noisy sentences. First of all, our system generates bi-gram pseudo sentences to solve the feature sparseness problem; they simply are made by combining two adjacent sentences by sliding window technique [9]. Then, since Title and Location methods show high performances, our system linearly combines these methods in the first step as follows:

$$
\operatorname{Score}\left(S_{i}\right)=\operatorname{sim}\left(S_{i}, Q\right)+\left(1-\frac{i-1}{N}\right) .
$$

where notations in this Equation follow those of Equation (1) and (3). After all the bigram pseudo sentences are scored by Equation (9), about $50 \%$ of them are removed because they are regarded as noisy sentences.

\subsubsection{Extracting Summary in the Second Step}

After the first step, the system can get more salient bi-gram pseudo sentences. Thereafter, it separates the bi-gram pseudo sentences remained from the first step into original single sentences. We here add Aggregation Similarity method to linear combination method of the first step (Equation (9)). Since noisy sentences are eliminated in the first step, the score of sentences could be improved as adding the Aggregation Similarity method; it considers the sum of similarities with other all sentences as the important score of a sentence. The final Equation is as follows:

$$
\operatorname{Score}\left(S_{i}\right)=\operatorname{sim}\left(S_{i}, Q\right)+\left(1-\frac{i-1}{N}\right)+w_{a} \operatorname{asim}\left(S_{i}\right) .
$$

where $w_{a}$ is a weight value reflecting the importance of Aggregation Similarity method. 
If documents have no title, TF-based query method is used instead of the Title method as shown in the following Equations (11) and (12).

$$
\begin{gathered}
\operatorname{Score}\left(S_{i}\right)=\operatorname{sim}\left(S_{i}, T f Q\right)+\left(1-\frac{i-1}{N}\right) . \\
\operatorname{Score}\left(S_{i}\right)=\operatorname{sim}\left(S_{i}, T f Q\right)+\left(1-\frac{i-1}{N}\right)+w_{a} \operatorname{asim}\left(S_{i}\right) .
\end{gathered}
$$

\section{Empirical Evaluation}

\subsection{Data Sets and Experimental Settings}

In our experiments, we used the summarization test set of KOrea Research and Development Information Center (KORDIC). This data is composed of news articles. The articles consist of several genres such as politics, culture, economics, and sports. Each test document has title, content, $30 \%$ summary, and $10 \%$ summary. The $30 \%$ and $10 \%$ summaries of the test document are made by manually extracting sentences from content. In our experiments, we used 841 document-summary pairs after eliminating duplicate articles and inadequate summary pairs although the size of summarization test set was reported as 1,000 documents [6].

We have two parameters to be determined; one of them is to adjust how many pseudo sentences are removed and the other is a weight parameter of Aggregation Similarity method in Equation (10), (12). For this parameter setting, we used 280 documents as a validation set which are selected at random. Hence, the test set in our experiments is composed of the rest of data set (561 documents).

To measure the performance of the method, F1 measure is used as the following Equation (13).

$$
F_{1}=\frac{2(P \times R)}{P+R}
$$

where $P$ is precision and $R$ is recall.

\subsection{Experimental Results}

\subsubsection{Comparing with One-Step Combination}

In order to verify the effectiveness of Two-step combination method, we compared the performance of Two-step combination method with that of One-step combination method. At first, our Two-step combination method without Aggregation Similarity method (2 step T\&L: Two-step Title and Location combination) reported higher performance than One-step combination method ( $T \& L$ : One-step Title and Location combination) as shown in Fig. 2. Next, to verify the addition of Aggregation Similarity method in the second step, we compared 2 step $T \& L$ and 2 step $T \& L \& A$ (Two-step Title, Location, and Aggregation Similarity combination). We also achieved the improved performance in 2 step $T \& L \& A$. Finally, we obtained $3.9 \%$ advanced score on the $10 \%$ summary and $1.5 \%$ advanced score on the $30 \%$ summary. 


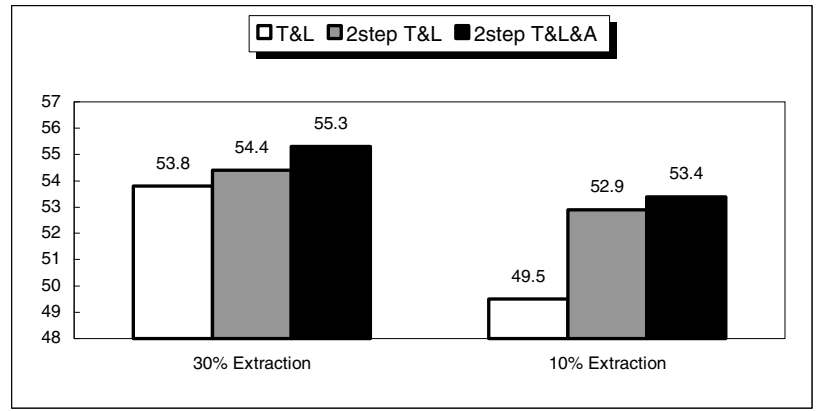

Fig. 2. Comparison between One-step and Two-step methods in case of using title

Table 3. Comparison between One-step and Two-step methods in case of using title

\begin{tabular}{cccc}
\hline & One-step & Two-step & Improvement \\
\hline $10 \%$ & 49.5 & 53.4 & $\mathbf{+ 3 . 9}$ \\
$30 \%$ & 53.8 & 55.3 & $\mathbf{+ 1 . 5}$ \\
\hline
\end{tabular}

In case of no-title, we used TF-based query method in place of Title method. In this cases, 2step $T f Q \& L \& A$ (Two-step TF-based query, Location and Aggregation Similarity combination) showed $1.1 \%$ and $2.3 \%$ advanced scores higher than $T f Q \& L$ (One-step TF-based query and Location combination) in $10 \%$ and $30 \%$ summary respectively as shown in Table 4 .

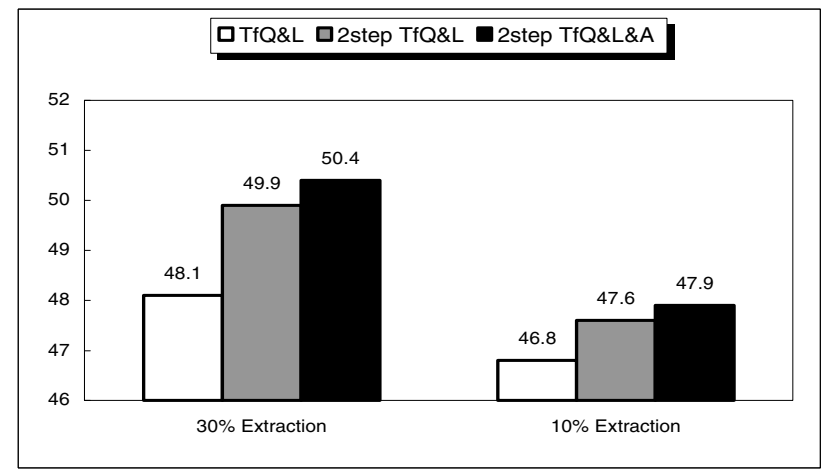

Fig. 3. Comparison between One-step and Two-step methods in case of using no-title

Table 4. Comparison between One-step and Two-step methods in case of using no-title

\begin{tabular}{cccc}
\hline & One-step & Two-step & Improvement \\
\hline $10 \%$ & 46.8 & 47.9 & $\mathbf{+ 1 . 1}$ \\
$30 \%$ & 48.1 & 50.4 & $\mathbf{+ 2 . 3 5}$ \\
\hline
\end{tabular}


Note that we chose $T \& L$ method for One-step because it showed the best performance in above both two cases (especially better than $T \& L \& A$ ).

\subsubsection{Comparing with Other Summarization Methods}

In this section, we make a comparison between our Two-step method and other systems such as Title, Location, and DOCUSUM. Especially, DOCUSUM is used for comparison with linguistic approaches.

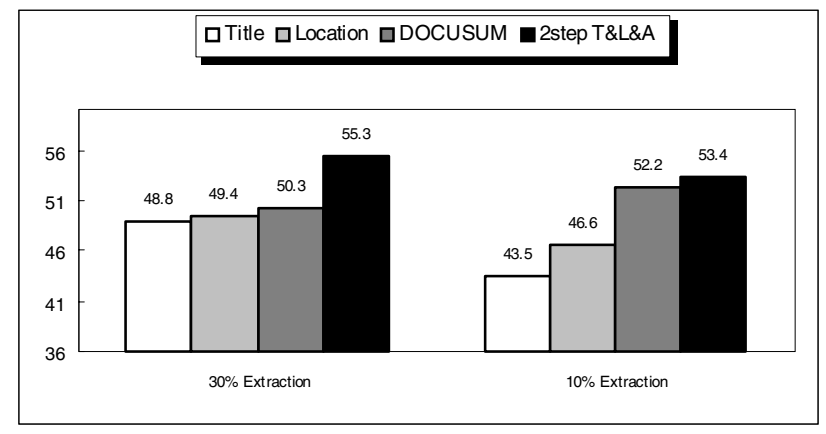

Fig. 4. Comparison between our method and other methods in case of using title

Table 5. Comparison between our method and other methods in case of using title

\begin{tabular}{cccc}
\hline & DOCUSUM & Two-step & Improvement \\
\hline $10 \%$ & 52.2 & 53.4 & $\mathbf{+ 1 . 2}$ \\
$30 \%$ & 50.3 & 55.3 & $\mathbf{+ 5 . 0}$ \\
\hline
\end{tabular}

As shown in Fig. 4 and Table 5, our system showed much better performance than title, location and even DOCUSUM. Even though DOCUSUM used knowledge resource such as context vector space for lexical clustering, it rather showed $1.2 \%$ and $5 \%$ lower performance than our method in $10 \%$ summary and $30 \%$ summary respectively.

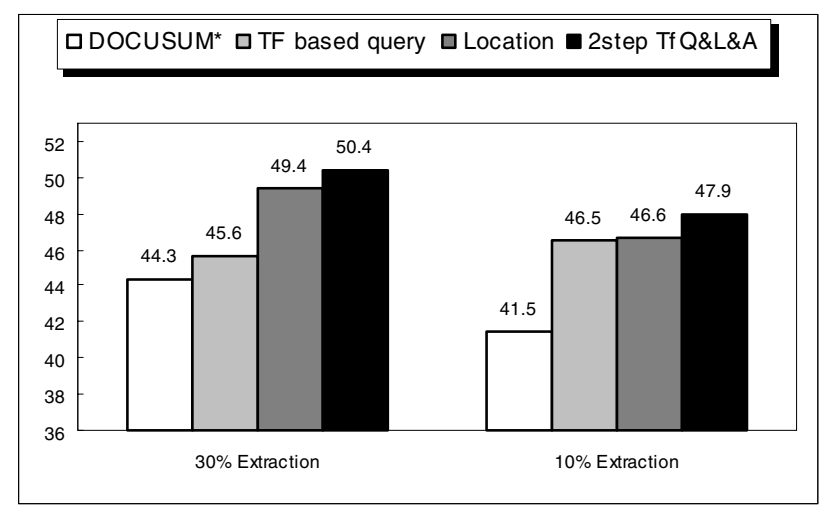

Fig. 5. Comparison between our method and other methods in case of using no-title 
Table 6. Comparison between our method and other methods in case of using no-title

\begin{tabular}{cccc}
\hline & DOCUSUM & Two-step & Improvement \\
\hline $10 \%$ & 41.5 & 47.9 & $\mathbf{+ 6 . 4}$ \\
$30 \%$ & 44.3 & 50.4 & $\mathbf{+ 6 . 1}$ \\
\hline
\end{tabular}

Moreover, we conducted experiments in no-title case. DOCUSUM* used only a topic keywords query without a title query extracted by its lexical clustering method. But the results in Fig. 5 also showed that our system performed much better than any other method (even DOCUSUM).

\section{Conclusions and Future Works}

In this paper, we have presented a new summarization method. It used bi-gram pseudo sentences to solve feature sparseness problem and Two-step combination method to improve the performance. As a result, we achieved higher performance than other statistical methods and DOCUSUM. Even though our system does not use any knowledge resource, it reported much better performance than DOCUSUM. In this paper, we implemented the high performance summarization system only to combine simple statistical methods on two steps. Our system has not only high performance but also the strong point to easily implement because it uses only simple statistical methods.

We plan to further researches on the multi-document summarization to apply our Two-step sentence extraction method to multi-document summarization. We will study the differentiation between single and multi-document summarization and implement new multi-document summarization system by using Two-step sentence extraction method.

\section{Acknowledgements}

This work was supported by grant No. R01-2003-000-11588-0 from the basic Research Program of the KOSEF and special research grant of Sogang University.

\section{References}

1. Barzilay, R., and Elhadad, M., Using Lexical Chains for Text Summarization, Advances in Automatic Summarization, The MIT Press (1999) 111-121.

2. Edmundson, H. P., New Methods in Automatic Extraction, Journal of the ACM 16(2) (1968) 264-285.

3. Goldstein, J., Kantrowitz, M., Mittal, V., and Carbonell, J., Summarizing Text Documents: Sentence Selection and Evaluation Metrics, In Proceedings of ACM-SIGIR'99 (1999) 121128

4. Han, K., Baek, D., and Rim, H., Automatic Text Summarization Based on Relevance Feedback with Query Splitting, In Proceedings of the Fifth International Workshop on Information Retrieval with Asian Languages (2000) 201-202. 
5. Kim, J. H., and Kim, J. H., Korean indicative summarization using aggregation similarity, In Proceedings of the 12th Conference on Hangul and Korean Language Information Processing, 238-244

6. Kim, T. H., Park, H. R., Shin, J. H., "Research on Text Understanding Model for IR/Summarization/Filtering", The Third Workshop on Software Science, Seoul, Korea. (1999)

7. Ko, Y., Kim, K., and Seo, J., Topic Keyword Identification for Text Summarization Using Lexical Clustering. IEICE transaction on information and system, Vol. E86-D, No. 9. (2003) 1695-1701.

8. Luhn, H. P., The Automatic Creation of Literature Abstracts, IBM Journal of Research and Development (1959) 159-165.

9. Maarek, Y., Berry, D., and Kaiser, G., 1991, An Information Retrieval Approach for Automatically Construction Software Libraries, IEEE Transaction on Software Engineering, Vol. 17, No. 8 (1991) 800-813.

10. Mani, I., Automatic Summarization, John Benjamins Publishing Co. (2001) 1-22.

11. Marcu, D., "Building up Rhetorical Structure Tree", In Proceedings of the 13th National Conference on Artificial Intelligence, Vol. 2 (1996) 1069 1074.

12. Miller, G., Beckwith, R., Fellbaum, C., Gross, D., and Miller, K., Introduction to WordNet: An on-line lexical database. International Journal of Lexicography (special issue) 3(4) (1990)234-245.

13. Salton, G., Automatic Text Processing: The Transformation, Analysis, and Retrieval of Information by Computer, Addison-Wesley Publishing Company (1989)

14. Wasson, M., Using leading text for news summaries: Evaluation results and implications for commercial summarization applications, In Proceedings of the 17th International Conference on Computational Linguistics and 36th Annual Meeting of the ACL, (1998) 13641368.

15. Zechner, K., Fast generation of abstracts from general domain text corpora by extracting relevant sentences, In Proceedings of the 16th international Conference on Computational Linguistics (1997) 986-989. 